INTERNATIONAL PHASE OF OCEAN DRILLING (IPOD)

DEEP SEA DRILLING PROJECT

DEVELOPMENT ENGINEERING

TECHNICAL NOTE NO. 6

\title{
OPERATIONAL TECHNICAL ACHIEVEMENTS
}




\section{DISCLAIMER}

This repart was prepared by the Deep Sea Drilling Prolect, University of Callfornla, San Dfego, as an account of work sponsored by the United States Government's National Science Foundation. Neither the University nor any of their employees, nor any of theit contractors, subcontractors, or their employees, makes any warranty, express or implied, or assumes any legal llablifty or responsibility for the accuracy, completeness or usefulness of any information, apparatus, product or process diselosed, or represents that its use would not infringe privately owned rights. 
TECHNICAL NOTE NO. 6

OPERATIONAL TECHNICAL ACHIEVEMENTS

\author{
Prepared for the \\ NATIONAL SCIENCE FOUNDATION \\ National Ocean Sediment Coring Program \\ Under Contract C-482 \\ by the \\ UNIVERSITY OF CALIFORNIA \\ Scripps Institution of Oceanography \\ Prime Contractor for the Project
}

June 1984

W. A. Nierenberg, Director

Scripps Institution of Oceanography
M. N. A. Peterson

Principal Investigator and Project Manager

Deep Sea Drilling Project

Scripps Institution of Oceanography 
The GLOMAR CHALLENGER was designed by Global Marine Inc. specifically for the earth science "Deep Sea Drilling Project." The ship was built in the Levingston Shipyards in 1966 to specifications developed by the Joint Oceanographic Institutions for Deep Earth Sampling and the National Science Foundation. The ship embarked on the first expedition (Leg 1) on August 11, 1968.

The GLOMAR CHALLENGER was the first dynamically positioned drill ship to operate in the deep oceans. Four (4) electrically powered thrusters (two in the bow and two in the stern) were used in combination with the ship's main propulsion to maintain station while drilling. A computer system was utilized to control the thruster and main propulsion output. The computer received a signal from sea floor beacons as to what repositioning of the ship was required.

Since the first expedition many new scientific tasks were assigned to the ship which were not originally anticipated. The majority were accomplished successfully and some were accomplished at the maximum capability of the CHALLENGER. Over the years the ship, the systems and components were redesigned or modified as required to accomplish the ever growing number of scientific experiments and tasks. 


\section{CONTENTS}

I TECHNICAL ACHIEVEMENTS $\ldots \ldots \ldots \ldots \ldots \ldots \ldots \ldots \ldots \ldots \ldots \ldots \ldots \ldots \ldots$

II PERFORMANCE AND TIME DISTRIBUTION $\ldots \ldots \ldots \ldots \ldots \ldots \ldots \ldots \ldots$

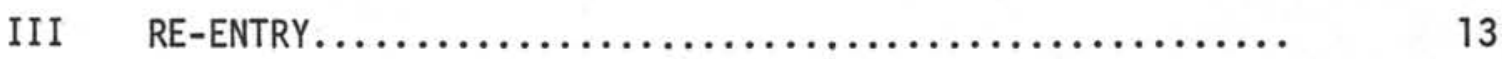

IV OPERATIONAL RECORDS/"TRIVIA" ACHIEVEMENTS $\ldots \ldots \ldots \ldots \ldots \ldots$ 
TECHNICAL ACHIEVEMENTS 
DRILLING AND CORING STATISTICS

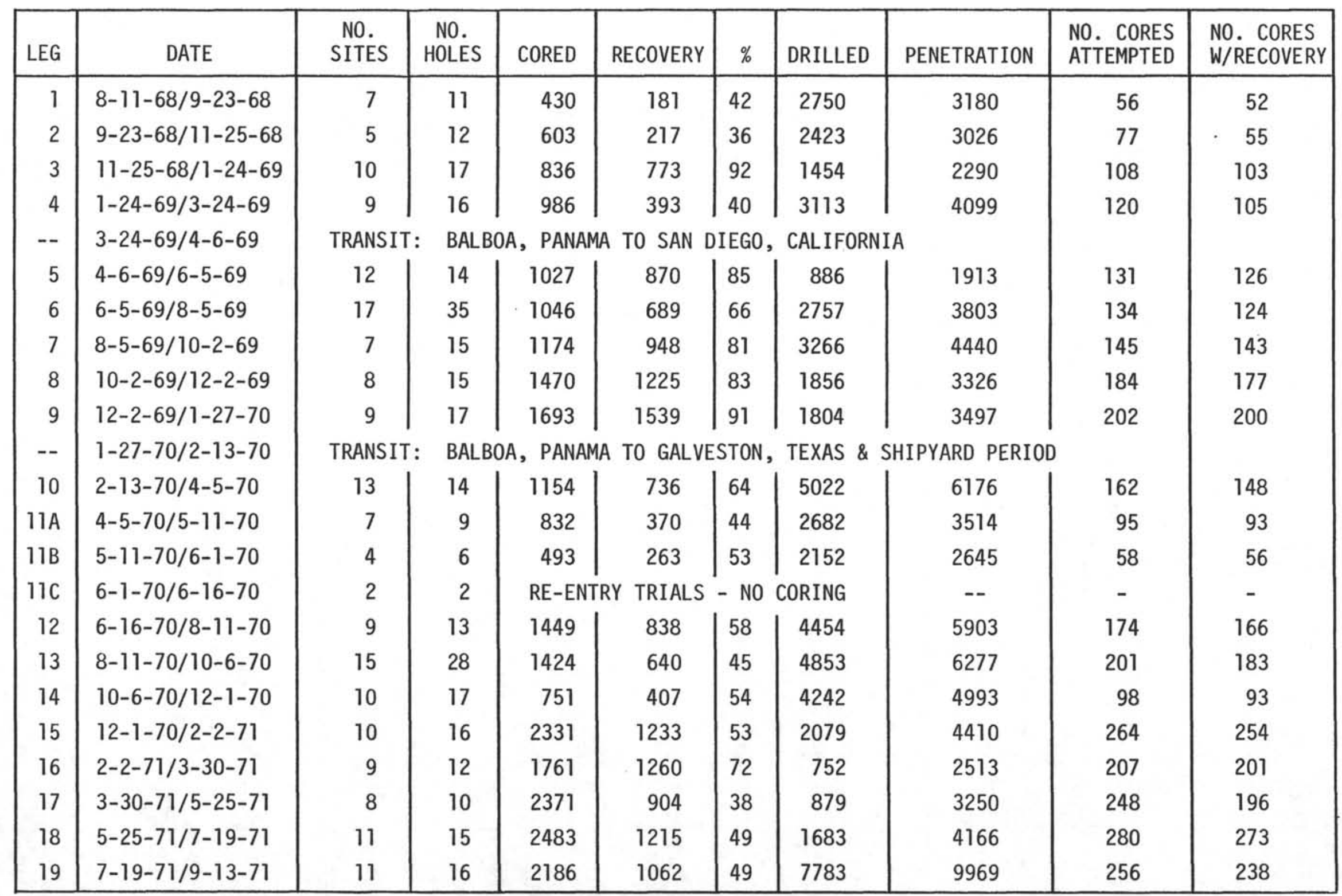

N

NOTE: CORING RESULTS IN METERS 
DRILLING AND CORING STATISTICS

(Continued)

\begin{tabular}{|c|c|c|c|c|c|c|c|c|c|c|}
\hline LEG & DATE & $\begin{array}{l}\text { NO. } \\
\text { SITES }\end{array}$ & $\begin{array}{l}\text { NO. } \\
\text { HOLES }\end{array}$ & CORED & RECOVERY & $\%$ & DRILLED & PENETRATION & $\begin{array}{l}\text { NO. CORES } \\
\text { ATTEMPTED }\end{array}$ & $\begin{array}{l}\text { N0. CORES } \\
\text { W/RECOVERY }\end{array}$ \\
\hline 20 & $9-13-71 / 11-10-71$ & 9 & 13 & 474 & 161 & 34 & 2698 & 3172 & 55 & 46 \\
\hline 21 & $11-10-71 / 1-11-72$ & 8 & 14 & 2574 & 1387 & 54 & 2133 & 4707 & 288 & 258 \\
\hline 22 & $1-11-72 / 3-6-72$ & 8 & 11 & 2543 & 1380 & 54 & 2090 & 4633 & 275 & 271 \\
\hline 23 & $3-6-72 / 5-1-72$ & 12 & 17 & 2594 & 1427 & 55 & 2808 & 5402 & 308 & 306 \\
\hline 24 & $5-1-72 / 6-26-72$ & 8 & 11 & 3194 & 1994 & 62 & 1260 & 4454 & 349 & 337 \\
\hline 25 & $6-26-72 / 8-22-72$ & 11 & 13 & 1394 & 790 & 57 & 2859 & 4253 & . 171 & 151 \\
\hline-- & $8-22-72 / 9-6-72$ & \multicolumn{9}{|c|}{ SHIPYARD PERIOD: DURBAN, SOUTH AFRICA } \\
\hline 26 & $9-6-72 / 10-30-72$ & 9 & 12 & 2234 & 1185 & 53 & 1659 & 3893 & 246 & 239 \\
\hline 27 & $10-30-72 / 12-9-72$ & 5 & 5 & 1571 & 960 & 61 & 874 & 2445 & 176 & 173 \\
\hline 28 & $12-9-72 / 2-27-73$ & 11 & 16 & 3014 & 1405 & 47 & 2633 & 5647 & 329 & 293 \\
\hline 29 & $2-27-73 / 4-18-73$ & 10 & 16 & 1909 & 1182 & 62 & 1290 & 3199 & 215 & 206 \\
\hline 30 & $4-18-73 / 6-13-73$ & 5 & 9 & 2332 & 1164 & 50 & 1808 & 4140 & 250 & 249 \\
\hline 31 & $6-13-73 / 8-4-73$ & 13 & 17 & 2612 & 1234 & 47 & 3661 & 6273 & 285 & 266 \\
\hline 32 & $8-4-73 / 10-10-73$ & 11 & 13 & 2211 & 739 & 33 & 1369 & 3580 & 241 & 220 \\
\hline 33 & $10-10-73 / 12-17-73$ & 5 & 8 & 1718 & 884 & 51 & 2748 & 4466 & 184 & 172 \\
\hline 34 & $12-17-73 / 2-3-74$ & 3 & 6 & 385 & 232 & 60 & 321 & 706 & 45 & 45 \\
\hline 35 & $2-3-74 / 3-30-74$ & 4 & 4 & 515 & 193 & 37 & 1697 & 2212 & 55 & 52 \\
\hline 36 & $3-30-74 / 5-22-74$ & 6 & 10 & 1006 & 579 & 58 & 1474 & 2480 & 107 & 102 \\
\hline 37 & $5-22-74 / 7-29-74$ & 4 & 9 & 1777 & 406 & 23 & 1415 & 3192 & 156 & 152 \\
\hline 38 & $7-29-74 / 9-25-74$ & 17 & 18 & 3237 & 1805 & 56 & 2042 & 5279 & 354 & 352 \\
\hline-- & $9-25-74 / 10-7-74$ & \multicolumn{9}{|c|}{ SHIPYARD PERIOD: AMSTERDAM } \\
\hline 39 & $10-7-74 / 12-17-74$ & 7 & 11 & 1535 & 1057 & 69 & 3126 & 4661 & 165 & 159 \\
\hline 40 & $12-17-74 / 2-15-75$ & 6 & 7 & 2339 & 1502 & 64 & 4190 & 6529 & 249 & 248 \\
\hline
\end{tabular}


DRILLING AND CORING STATISTICS

(Continued)

\begin{tabular}{|r|l|r|r|r|r|r|r|r|r|r|}
\hline LEG & \multicolumn{1}{|c|}{ DATE } & $\begin{array}{c}\text { NO. } \\
\text { SITES }\end{array}$ & $\begin{array}{c}\text { NO. } \\
\text { HOLES }\end{array}$ & CORED & RECOVERY & $\%$ & DRILLED & PENETRATION & $\begin{array}{c}\text { NO. CORES } \\
\text { ATTEMPTED }\end{array}$ & $\begin{array}{c}\text { NO. CORES } \\
\text { W/RECOVERY }\end{array}$ \\
\hline 41 & $2-15-75 / 4-10-75$ & 5 & 7 & 2786 & 1673 & 60 & 2276 & 5062 & 300 & 295 \\
$42 A$ & $4-10-75 / 5-21-75$ & 8 & 11 & 1192 & 670 & 56 & 3269 & 4461 & 150 & 144 \\
$42 B$ & $5-21-75 / 6-11-75$ & 3 & 6 & 2318 & 1275 & 55 & 420 & 2738 & 251 & 242 \\
43 & $6-11-75 / 8-12-75$ & 6 & 6 & 1769 & 958 & 54 & 1363 & 3132 & 189 & 184 \\
44 & $8-12-75 / 9-30-75$ & 5 & 11 & 1343 & 547 & 41 & 2024 & 3367 & 149 & 142 \\
-- & $9-30-75 / 11-8-75$ & SHIPYARD & IPOD MOBILIZATION PERIOD: & NORFOLK, VA. & & & & \\
$44 \mathrm{~A}$ & $11-8-75 / 11-27-75$ & 2 & 4 & 75 & 31 & 41 & 545 & 620 & 8 & 8 \\
45 & $11-27-75 / 1-20-76$ & 2 & 3 & 994 & 328 & 33 & 76 & 1070 & 112 & 108 \\
46 & $1-20-76 / 3-10-76$ & $0 *$ & 2 & 283 & 64 & 23 & 245 & 528 & 35 & 32 \\
47 & $3-10-76 / 5-12-76$ & 2 & 7 & 2810 & 1813 & 65 & 2040 & 4850 & 301 & 292 \\
48 & $5-12-76 / 7-13-76$ & 8 & 10 & 2996 & 1230 & 41 & 996 & 3992 & 318 & 308 \\
49 & $7-13-76 / 9-4-76$ & 8 & 11 & 1944 & 881 & 45 & 736 & 2680 & 207 & 202 \\
50 & $9-4-76 / 11-10-76$ & 2 & 5 & 821 & 358 & 44 & 2355 & 3176 & 80 & 78 \\
$51 \mathrm{~A}$ & $11-10-76 / 12-13-76$ & 1 & 2 & 430 & 252 & 59 & 113 & 543 & 48 & 44 \\
$51 \mathrm{~B}$ & $12-13-76 / 1-17-77$ & $0 *$ & 3 & 372 & 209 & 56 & 211 & 583 & 48 & 44 \\
52 & $1-17-77 / 3-9-77$ & 1 & $2 *$ & 566 & 335 & 59 & 187 & 753 & 71 & 71 \\
53 & $3-9-77 / 4-25-77$ & $0 *$ & 1 & 627 & 406 & 65 & 0 & 627 & 73 & 72 \\
54 & $4-25-77 / 6-18-77$ & 11 & 18 & 847 & 461 & 54 & 435 & 1282 & 103 & 102 \\
-- & $6-18-77 / 7-13-77$ & SHIPYARD PERIOD: & TERMINAL ISLAND, CALIFORNIA & & & \\
55 & $7-13-77 / 9-6-77$ & 4 & 11 & 807 & 405 & 50 & 424 & 1231 & 104 & 96 \\
56 & $9-6-77 / 10-10-77$ & 4 & 7 & 1323 & 497 & 38 & 736 & 2059 & 142 & 135 \\
57 & $10-10-77 / 12-4-77$ & 4 & 10 & 2524 & 1424 & 56 & 3309 & 5833 & 273 & 262 \\
58 & $12-4-77 / 1-30-78$ & 5 & 9 & 2972 & 1592 & 54 & 722 & 3694 & 324 & 322 \\
\hline
\end{tabular}


DRILLING AND CORING STATISTICS

(Continued)

\begin{tabular}{|c|c|c|c|c|c|c|c|c|c|c|}
\hline LEG & DATE & $\begin{array}{l}\text { NO. } \\
\text { SITES }\end{array}$ & $\begin{array}{l}\text { NO. } \\
\text { HOLES }\end{array}$ & CORED & RECOVERY & $\%$ & DRILLED & PENETRATION & $\begin{array}{l}\text { N0. CORES } \\
\text { ATTEMPTED }\end{array}$ & $\begin{array}{l}\text { NO. CORES } \\
\text { W/RECOVERY }\end{array}$ \\
\hline 59 & $1-30-78 / 3-15-78$ & 5 & 7 & 2779 & 1162 & 42 & 428 & 3227 & 322 & 306 \\
\hline 61 & $5-15-78 / 7-29-78$ & 1 & 2 & 1246 & 726 & 58 & 440 & 1686 & 161 & 156 \\
\hline 62 & $7-29-78 / 9-6-78$ & 4 & 5 & 1976 & 635 & 32 & 39 & 2015 & 218 & 215 \\
\hline 64 & $11-27-78 / 1-14-79$ & 8 & 14 & 2936 & 1631 & 56 & 424 & 3360 & 344 & 322 \\
\hline 65 & $1-14-79 / 3-13-79$ & 4 & 15 & 1323 & 750 & 57 & 680 & 2003 & 179 & 172 \\
\hline 66 & $3-13-79 / 5-2-79$ & 8 & 14 & 3155 & 1844 & 58 & 456 & 3611 & 353 & 351 \\
\hline 67 & $5-2-79 / 6-27-79$ & 7 & 15 & 2316 & 1182 & 51 & 1147 & 3463 & 254 & 243 \\
\hline 69 & $9-18-79 / 10-29-79$ & 2 & 7 & 784 & 455 & 58 & 1061 & 1851 & 125 & 123 \\
\hline 70 & $10-29-79 / 12-28-79$ & $5 *$ & 32 & 817 & 478 & 59 & 192 & 1009 & 151 & 148 \\
\hline 71 & $12-28-79 / 2-21-80$ & 4 & 6 & 1303 & 822 & 63 & 139 & 1442 & 173 & 163 \\
\hline 72 & $2-21-80 / 4-8-80$ & 4 & 12 & 2163 & 1544 & 71 & 550 & 2713 & 308 & 297 \\
\hline 73 & $4-8-80 / 6-1-80$ & 6 & 13 & 1475 & 1051 & 71 & 583 & 2058 & 286 & 281 \\
\hline 74 & $6-1-80 / 7-24-80$ & 5 & 11 & 2573 & 1830 & 71 & 566 & 3139 & 356 & 249 \\
\hline 75 & $7-24-80 / 9-6-80$ & 3 & 8 & 1931 & 1445 & 75 & 239 & 2170 & 342 & 328 \\
\hline-- & $9-6-80 / 10-11-80$ & TRANSIT & \multicolumn{8}{|c|}{ RECIFE, BRAZIL TO NORFOLK, VA. \& SHIPYARD PERIOD } \\
\hline 76 & $10-11-80 / 12-21-80$ & 2 & 4 & 1532 & 985 & 64 & 762 & 2294 & 202 & 199 \\
\hline
\end{tabular}


DRILLING AND CORING STATISTICS

(Continued)

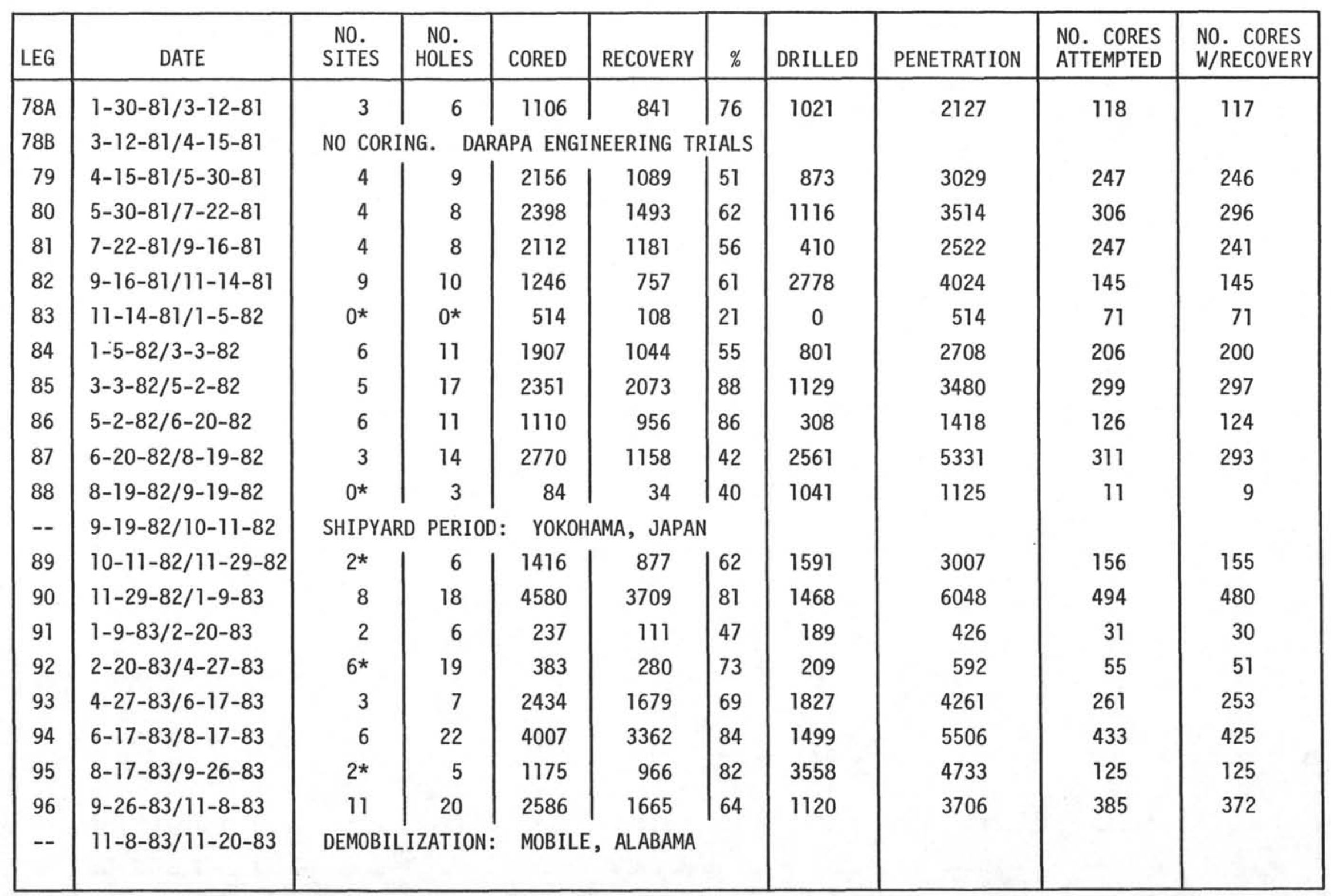

or

* RETURNED tO SITE AND/OR hOLE AS INDICATED 
INTERNATIONAL PHASE OF OCEAN DRILLING

DEEP SEA DRILLING PROJECT

TECHNICAL ACHIEVEMENT AFTER 96 CRUISES

AUGUST 11, 1968 - NOVEMBER 20, 1983

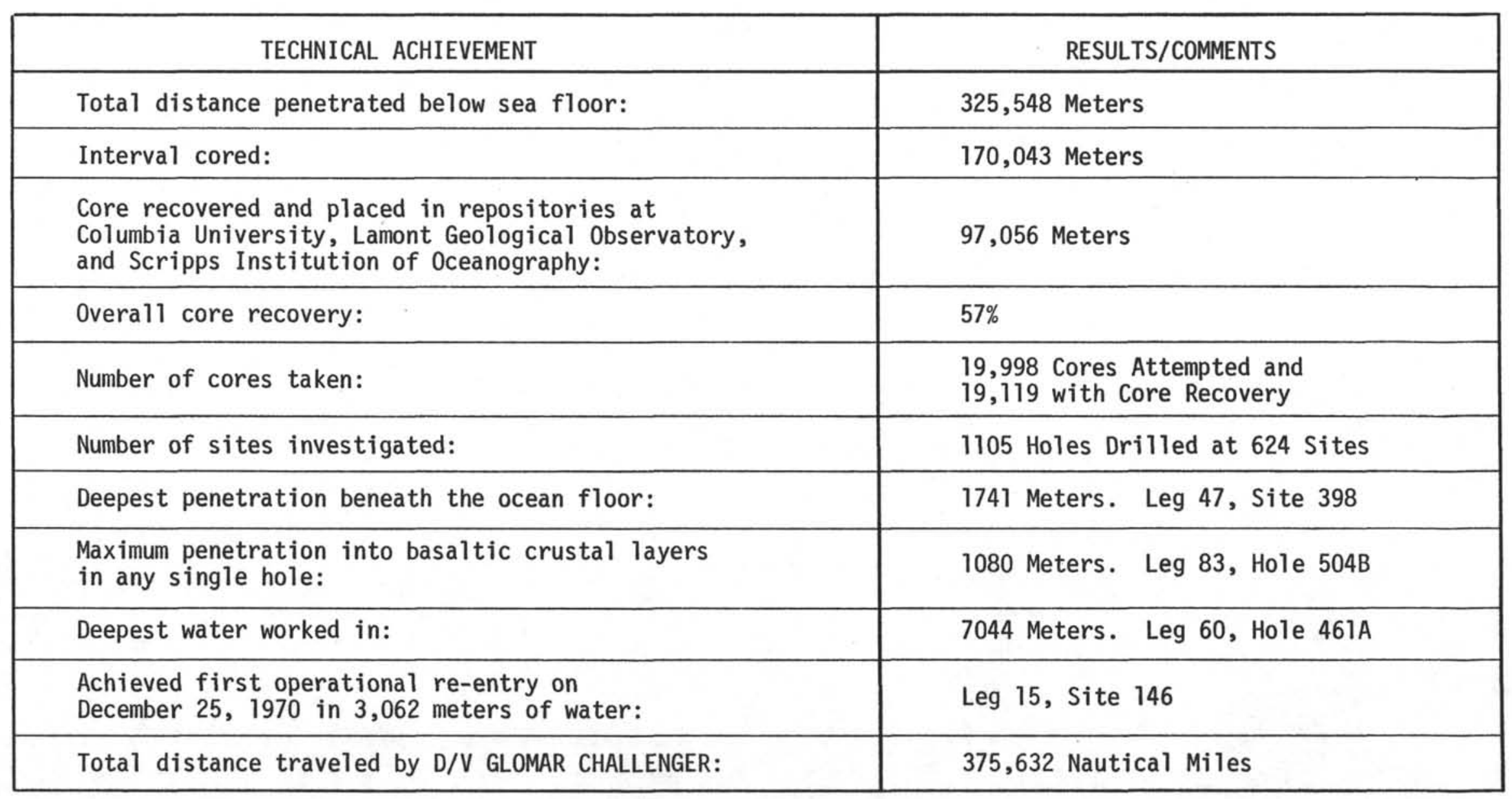




\section{INSTRUMENT EMPLACEMENTS}

1. HIG - LEG 64 SITE 482

2. HIG - LEG 67 SITE 494

3. HIG - LEG 78A SITE 543. INSTRUMENT RETRIEVED DUE TO EQUIPMENT PROBLEMS

4. DARPA - LEG 78B SITE 395. INSTRUMENT IMPLANTED AND RETRIEVED

5. HIG - LEG 88 SITE 581

6. DARPA - LEG 91 SITE 595

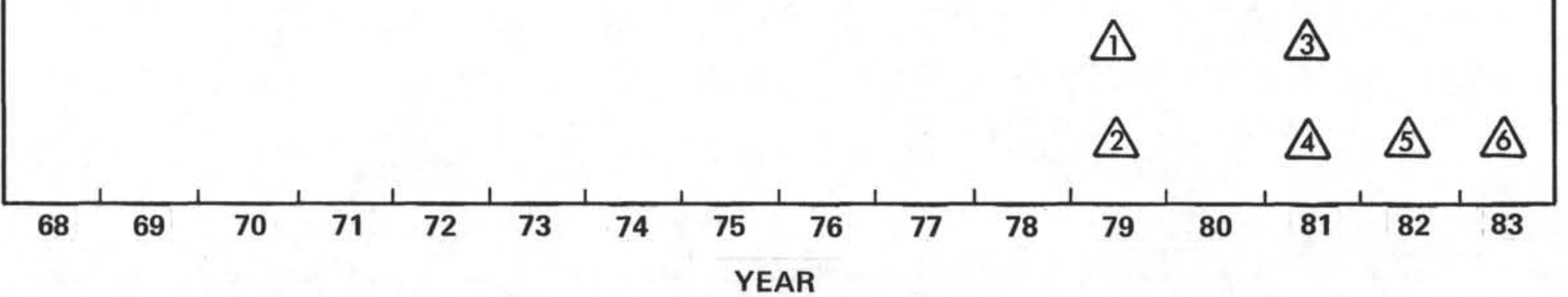


PERFORMANCE AND TIME DISTRIBUTION 
Following are performance figures for D/V GLOMAR CHALLENGER from beginning of Leg 1, August 11, 1968 to the end of the drilling contract November 20, 1983.

133,920 hours recorded since DSDP became operational.

70,099 hours or $52.3 \%$ of total time spent on site.

4,010 hours or $3 \%$ recorded as total equipment breakdown.

1,347 hours or $1 \%$ downtime due to inoperative weather conditions.

15,108 hours or $11.3 \%$ spent in port calls and demobilization.

43,356 hours or $32.4 \%$ spent cruising from site to site and into/out of port. 
GLOMAR CHALLENGER

PERFORMANCE RECORD

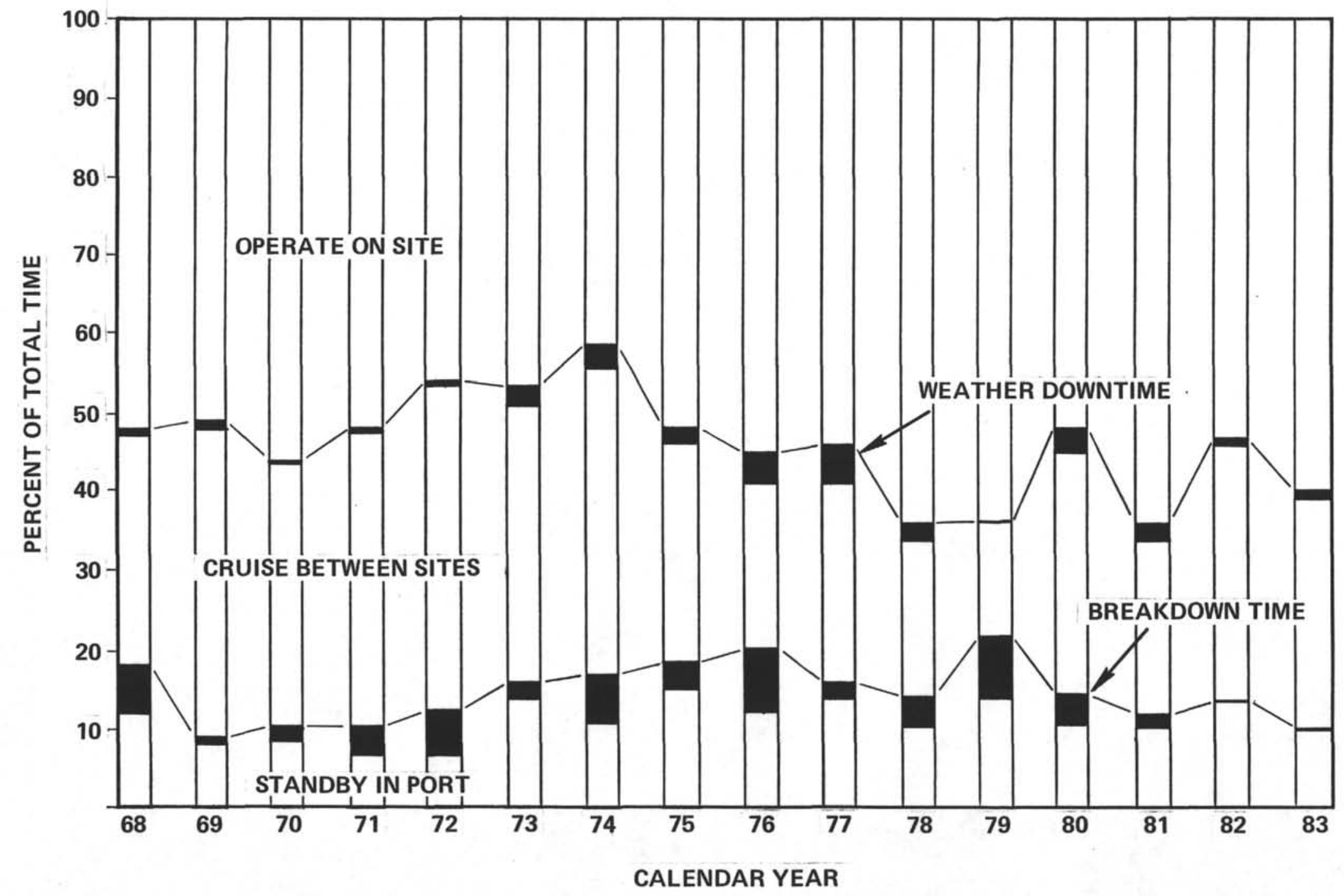




\section{RE-ENTRY}

Engineering trials were conducted on Leg $11 \mathrm{C}$ in June, 1970 and the concept of re-entry was proven to be feasible. The first operational re-entry was achieved in December, 1970 on Leg 15 at Site 146.

Four (4) re-entries were achieved in 1973 on Legs 30 and 34 . The equipment was removed from the ship and major modifications were made by the EDO Western Corporation and Deep Sea Drilling. During Leg 37, ten (10) re-entries were made, nine (9) on Site 332 and one (1) at Site 333.

Equipment was again removed and modified/updated for the upcoming DSDP/IPOD Ocean Drilling Phase. Commencing with Leg 45, December, 1975 until November, 1983 a total of 146 re-entries were made at 23 separate sites.

EDO Western is to be commended for their effort and interest during re-entry operations. Without this, the re-entry success would have been impossible to achieve. DSDP personnel should be proud to have been part of this program. The record and achievements speak for themselves. 
SUMMARY OF RE-ENTRY

\begin{tabular}{|c|c|c|c|}
\hline$\underline{\text { LEG }}$ & SITE & $\begin{array}{c}\text { (METERS) } \\
\text { WATER DEPTH } \\
\end{array}$ & $\begin{array}{l}\text { NUMBER OF } \\
\text { RE-ENTRIES } \\
\end{array}$ \\
\hline 15 & 146 & $3939 \mathrm{~m}$ & 2 \\
\hline 30 & $288 \mathrm{~A}$ & $3030 \mathrm{~m}$ & 2 \\
\hline 34 & $319 A$ & $4296 \mathrm{~m}$ & 1 \\
\hline 34 & $320 B$ & $4487 \mathrm{~m}$ & 1 \\
\hline 37 & $332 B$ & $1841 \mathrm{~m}$ & 9 \\
\hline 37 & 333 & $1682 \mathrm{~m}$ & 1 \\
\hline $45 / 78 B$ & $395 A$ & $4485 \mathrm{~m}$ & 13 \\
\hline 46 & $396 B$ & $4465 \mathrm{~m}$ & 7 \\
\hline 47 & $398 D$ & $3900 \mathrm{~m}$ & 2 \\
\hline 48 & $400 \mathrm{~A}$ & $4399 \mathrm{~m}$ & 1 \\
\hline 50 & $415 A$ & $2817 \mathrm{~m}$ & 3 \\
\hline 50 & $416 \mathrm{~A}$ & $4203 \mathrm{~m}$ & 9 \\
\hline $51 B / 52$ & 4170 & $5489 \mathrm{~m}$ & 10 \\
\hline $52 / 53$ & $418 \mathrm{~A}$ & $5519 \mathrm{~m}$ & 13 \\
\hline 55 & $433 C$ & $1874 \mathrm{~m}$ & 3 \\
\hline 57 & $438 B$ & $1575 \mathrm{~m}$ & 1 \\
\hline 58 & $442 B$ & $4645 \mathrm{~m}$ & 2 \\
\hline $61 / 89$ & $462 A$ & $5186 \mathrm{~m}$ & 19 \\
\hline 65 & 482D & $3012 \mathrm{~m}$ & 1 \\
\hline 65 & $483 B$ & $3084 \mathrm{~m}$ & 7 \\
\hline 69 & $504 A$ & $3468 \mathrm{~m}$ & 2 \\
\hline $69 / 70 / 83 / 92$ & $504 B$ & $3474 \mathrm{~m}$ & 27 \\
\hline 76 & $534 \mathrm{~A}$ & $4976 \mathrm{~m}$ & 8 \\
\hline 79 & $547 \mathrm{~B}$ & 3952 m & 3 \\
\hline 81 & 553 & $2339 \mathrm{~m}$ & 2 \\
\hline 88 & $581 \mathrm{~B}$ & $5478 \mathrm{~m}$ & 2 \\
\hline 91 & $595 B$ & $5629 \mathrm{~m}$ & 3 \\
\hline 92 & $597 C$ & $4157 \mathrm{~m}$ & 2 \\
\hline 93 & $603 B$ & $4644 \mathrm{~m}$ & 4 \\
\hline
\end{tabular}




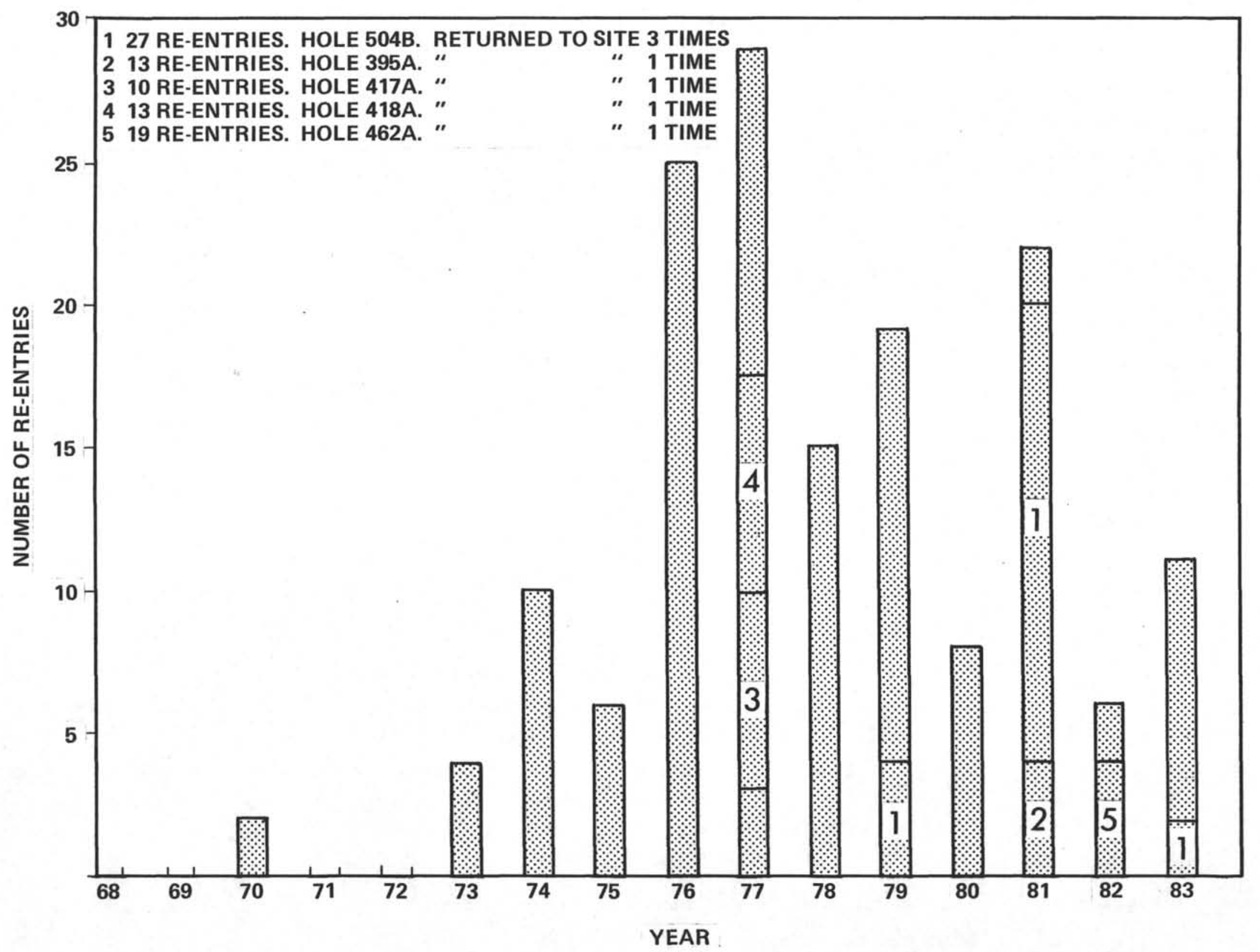




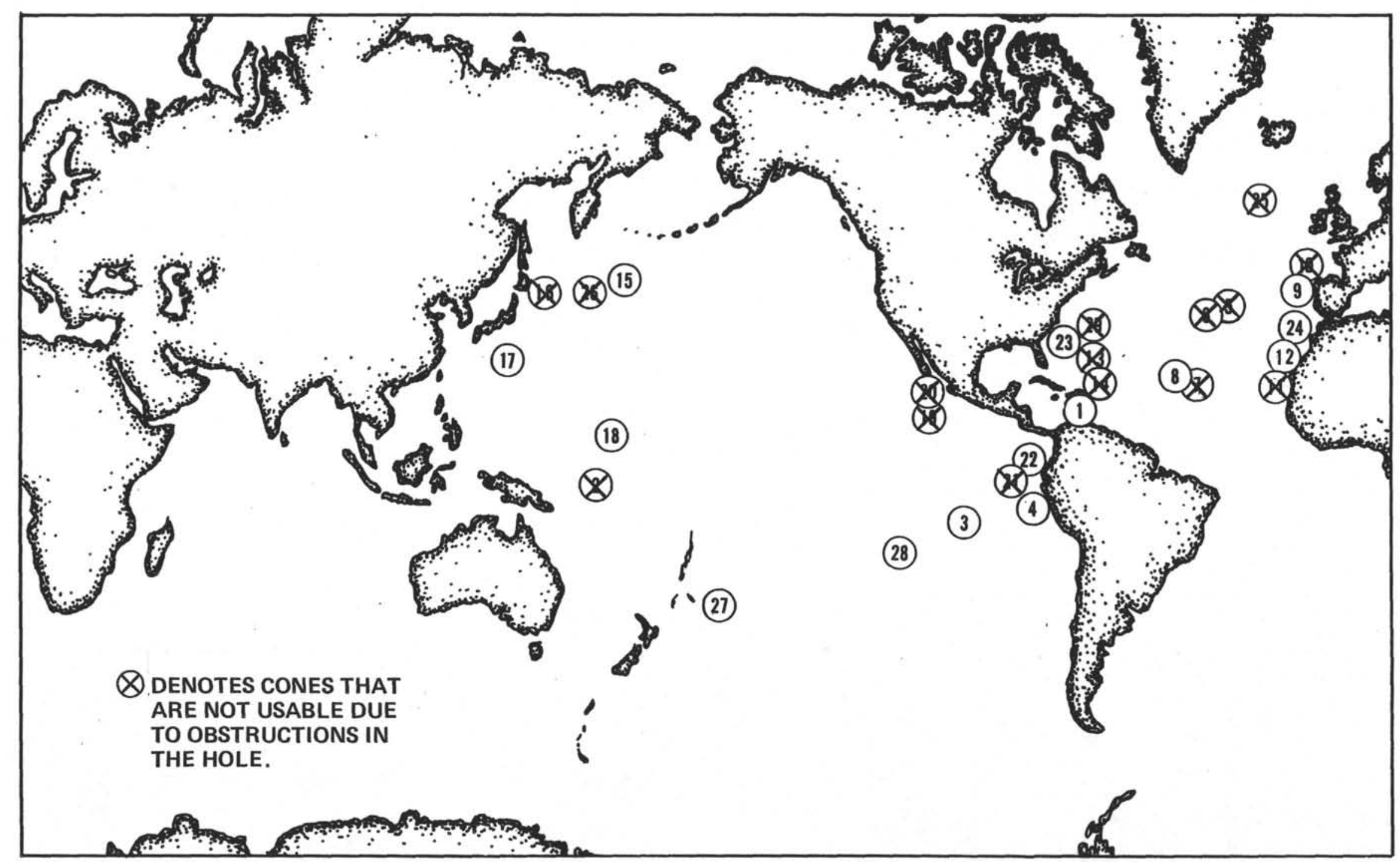

RE-ENTRY CONE DEPLOYMENT MAP 
OPERATIONAL RECORDS/"TRIVIA" ACHIEVEMENTS 
DSDP OPERATIONAL RECORDS

NOVEMBER 20, 1983

THROUGH LEG 96

\begin{tabular}{|c|c|c|c|c|}
\hline & & $\underline{\text { LEG }}$ & SITE & HOLE \\
\hline Longest GMI Leg & 71.7 days & 74 & & \\
\hline Longest Science Staff Leg & 83.3 days & 39 & & \\
\hline Shortest Leg & 34.1 days & 56 & & \\
\hline Longest Distance Traveled & 9895.0 n.m. & 39 & & \\
\hline Shortest Distance Traveled & 813.7 n.m. & 93 & & \\
\hline Greatest Average Speed & $10.7 \mathrm{kt}$ & 3 & & \\
\hline Highest Percentage of Site Time & $80.0 \%$ & 57 & & \\
\hline Most Site Time On One Leg & 51.8 days & 61 & & \\
\hline Most Time On Site & 83.0 days & $69 / 70 / 83$ & 504 & $504,504 A, B$ \\
\hline Most Time On One Hole & 73.0 days & $69 / 70 / 83$ & 504 & $504 \mathrm{~B}$ \\
\hline Most Sites Investigated & 17 & $6 / 38$ Tie & & \\
\hline Most Holes Drilled & 36 & 6 & & \\
\hline Most Holes On One Site & 10 & 70 & $506 / 507$ & \\
\hline Shallowest Water Operations Attempted & $197.0 \mathrm{~m}$ & 18 & 176 & \\
\hline Shallowest Water Operations Successful & $505.0 \mathrm{~m}$ & 28 & 273 & \\
\hline Deepest Water Operations & $7044.0 \mathrm{~m}$ & 60 & 461 & $461 \mathrm{~A}$ \\
\hline Longest Drill String & $7059.5 \mathrm{~m}$ & 60 & 461 & $461 / 461 \mathrm{~A}$ \\
\hline Deepest Penetration, Multiple Bit & $1740.0 \mathrm{~m}$ & 47 & 398 & $398 D$ \\
\hline
\end{tabular}


DSDP OPERATIONAL RECORDS

NOVEMBER 20, 1983

THROUGH LEG 96

(Continued)

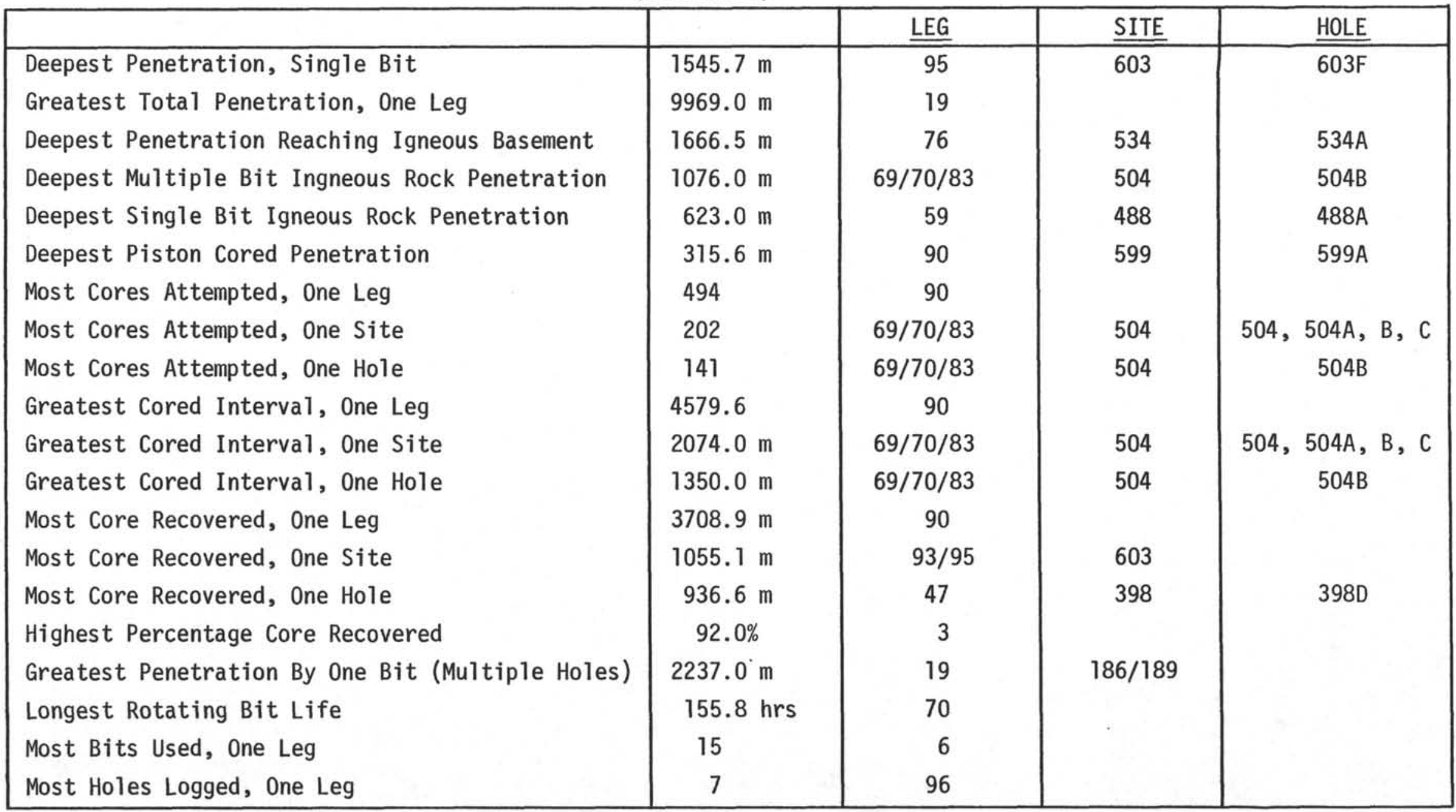


DSDP OPERATIONAL RECORDS

NOVEMBER 20, 1983

THROUGH LEG 96

(Continued)

\begin{tabular}{|l|c|c|c|c|}
\hline & & $\underline{\text { LEG }}$ & SITE & HOLE \\
\hline Most Logs Run, One Leg & 28 & 64 & & \\
Longest Continuous Open-Hole Log Interval & $1073.9 \mathrm{~m}$ & 83 & 504 & $504 \mathrm{~B}$ \\
First Successful Logging & & 4 & & \\
First Re-entry & & $11 \mathrm{C}$ & & \\
First Operational Re-entry & 16 & 15 & & \\
Most Re-entries, One Leg & 27 & $69 / 70 / 83 / 92$ & 504 & $504 \mathrm{~B}$ \\
Most Re-entries, One Hole & $5630.0 \mathrm{~m}$ & 91 & 595 & $504 \mathrm{~B}$ \\
Deepest Water Re-entry & $2.0 \mathrm{~min}$. & 83 & 504 & $504 \mathrm{~B}$ \\
Fastest Re-entry Scan Time & & & & \\
\hline
\end{tabular}

